Available online on 15.01 .2020 at http://jddtonline.info
Open Access to Pharmaceutical and Medical Research
unrestricted non-commercial use, provided the original work is properly cited

Open 1 Access

Research Article

\title{
Ligand Decorated Embelin Loaded PLGA Nanoparticles for Management of Alcohol Induced Hepatotoxicity
}

\author{
Kumar Ajay ${ }^{1}$, Khan M. Nikhat ${ }^{2}$, Kanoujia Jovita ${ }^{3}$, Singh Amandeep ${ }^{2}$, Mishra Neeraj ${ }^{3 *}$ \\ ${ }^{1}$ Government Pharmacy Institute, Agamkuan, Patna, 800007, India \\ ${ }^{2}$ Department of Pharmaceutics, I.S.F. College of Pharmacy, GhalKalan, Ferozpur G.T. Road, Moga, Punjab, India; 142001 \\ ${ }^{3}$ Amity Institute of Pharmacy, Amity University Madhya Pradesh, Gwalior, India
}

\begin{abstract}
Preparation of surface modified Embelin loaded nanoparticles (GA-PEG-PLGA) for the management of hepatotoxicity. Surface modified Embelin loaded GA-PEG-PLGA NPs were evaluated by NMR, FTIR, TEM techniques and in vitro release studies. The biodistribution of the nanoparticles was assessed by High-performance liquid chromatography (HPLC), and the cellular uptake study was evaluated using Hep G2 cells (liver cells lines). The hepatoprotecttive effect of the surface modified Embelin loaded GA-PEG-PLGA NPs was investigated in-vitro and in-vivo. The surface modified Embelin loaded GA-PEG-PLGA nanoparticles significantly increases the uptake of drug in liver by 2.5 folds more than plain drug.
\end{abstract}

Keywords: Glycyrrhetinic acid, Receptor mediated, Surface functionalization, Embelin, PLGA nanoparticle.

Article Info: Received 19 Nov 2019; Review Completed 28 Dec 2019; Accepted 06 Jan 2020; Available online 15 Jan 2020

Cite this article as:

Kumar A, Khan MN, Kanoujia J, Singh A, Mishra N, Ligand Decorated Embelin Loaded PLGA Nanoparticles for Management of Alcohol Induced Hepatotoxicity, Journal of Drug Delivery and Therapeutics. 2020; 10(1):72-80

http://dx.doi.org/10.22270/jddt.v10i1.3844

口ir. *Address for Correspondence:

Dr. Neeraj Mishra, Amity Institute of Pharmacy, Amity University of Madhya Pradesh, Maharajpura (Opposite Airport), Gwalior-474005 (M.P.)

\section{INTRODUCTION}

Liver, the largest gland of the body, is a vital organ. It is an important and complex organ, located in the right upper quadrant of abdomen. Its functions including detoxification of chemicals, metabolism of drugs, protein synthesis, and the production of biological chemicals, are necessary for the conservation of life. Thus, the patients with long term liver diseases such as hepatitis, liver fibrosis, and hepatic cancer need immediate attention to sustain life, and as a result are often exposed to the prolonged treatment with drugs (1). But the liver is the site for drug detoxification and biotransformation of exogenous chemicals that penetrate body systems via distinct routes (2). Therefore it is very important to seek new therapeutic strategies to treat liver diseases. Targeted drug delivery, which can carry the drugs to specific organs or tissues, is a highly desirable strategy to improve the therapeutic outcome, with significantly decreased toxic side-effects compared to traditional chemotherapy $(3,4)$. It is the metabolic "engine room of the body". Embelin (2, 5-dihydroxy-3-undecyl-1, 4 benzoquinone) is a naturally occurring alkyl benzoquinone, the active principle in the fruits of Embelia ribes belonging to family Myrsinaceae. Embelin have been reported for the treatment of various liver diseases like jaundice $(5,6)$. Embelin protects the liver injury caused by alcohol administration. In view of its nontoxic nature, it may be developed as an effective therapeutic agent for alcoholinduced liver disease by its antioxidant and antiinflammatory features $(7,8)$. Accumulated evidence has demonstrated that both oxidative stress and abnormal cytokine production, especially tumor necrosis factor- $\alpha$ (TNF), play important etiological roles in the pathogenesis of alcoholic liver disease (ALD). Agents that have both antioxidant and anti-inflammation properties, particularly anti-TNF production, represent promising therapeutic interventions for $\operatorname{ALD}(5,9)$. We investigated the effects and the possible mechanism of Embelin on liver injury induced by alcohol administration.

Abundantly available receptor, which is specific to hepatic parenchymal cells is asialoglycoprotein receptor. It has the ability to recognize the galactosylated ligands, lactobionic acid ligand, asialofetuin ligands, soybean derived steryglyucoside (SG) ligands and many more. Similarly, glycyrrhetinic acid receptors are mainly found on the sinusiodal surface of mammalian hepatocytes. It has ability to recognize the glycyrrrhetinic acid ligands (10). 
Glycyrrhetinic acid (GA) is a hydrolysis product of glycyrrhizic acid (GL), which is the main component of the Traditional Chinese Medicine liquorice. It was reported that there are some highly specific GA binding sites located on the surface of liver parenchyma cells (11).The investigations of GA-mediated targeted drug delivery systems such as liposomes, nanoparticles and polymeric micelles have become more and more popular. Wei Huang was prepared nanoparticles of paclitaxel by using glycyrrhetinic acid as targeting ligand and hyalouronic acid as polymer (12). Prepared nanoparticles showed high efficiency upto $31.16 \%$ and entrapment efficiency of $92.02 \%$. The prepared conjugate showed high liver targeting as compared to the other organs. Hiraku Onishi was prepared Poly (L-lactic acidco-glycolic acid) with glycyrrhetic acid-containing microparticles as dosage forms for liver-specific delivery of a liver-protective drug (13). Zhi Yuan was prepared nanoparticles with PLGA and Glycyrrhetinic acid-mediated nanoparticles of hepatic targeted drug delivery system (14). In our study, Embelin is formulated as a hepatoprotective agent for liver targeting. Aim of the present study was to design surface modified PLGA nanoparticles combined with an active ligand (GA) to achieve the desired targeting performance. The main aim of the study is to make embelin loaded glycyrrhetinic acid modified PLGA nanoparticles as novel hepatic drug delivery system and prepared formulation was further evaluated for serum glutamic oxaloacetic transaminase (SGOT) and serum glutamic pyruvic transaminase (SGPT), alkaline phosphate (ALP) and total bilirubin (TB) assessment of liver function.

\section{MATERIALS \& METHODS}

PLGA (MW 8000 Da, Copolymer ratio 50:50) and Bis amine PEG were obtained from Sigma-Aldrich, Dialysis membrane (MWCO 5, 10, 12 and 14 KD) were purchased from Himedia labs, (India). Glycyrrhetinic Acid, Dicyclohexylcarbodiimide (DCC) and N-hydroxy-succinimide (NHS) were obtained from Himedia Pvt Ltd, Embelin was obtained from Indofine chemical company, Inc, USA and Hep G2 cell lines were obtained from National Centre for Cell Sciences (NCCS). All other chemicals were used of extra pure grade.

\subsection{Synthesis of Glycyrrhetinic Acid -PEG-PLGA Conjugate}

The GA-PEG-PLGA conjugate was synthesized in four steps, i.e. glycyrrhetinic acid (GA) activation, PLGA activation, synthesis of GA-PEG- $\mathrm{NH}_{2}$, and final synthesis of GA-PEGPLGA conjugate as depicted in Fig 1.

\section{Activation of Glycyrrhetinic acid}
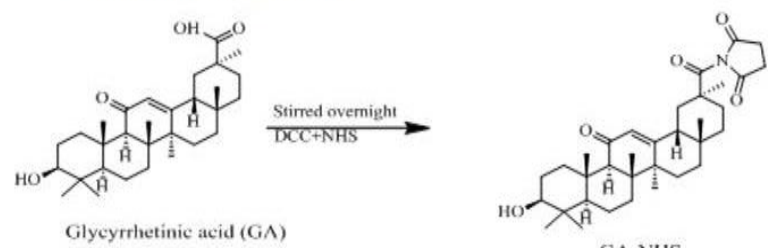

2. Activation of PLGA
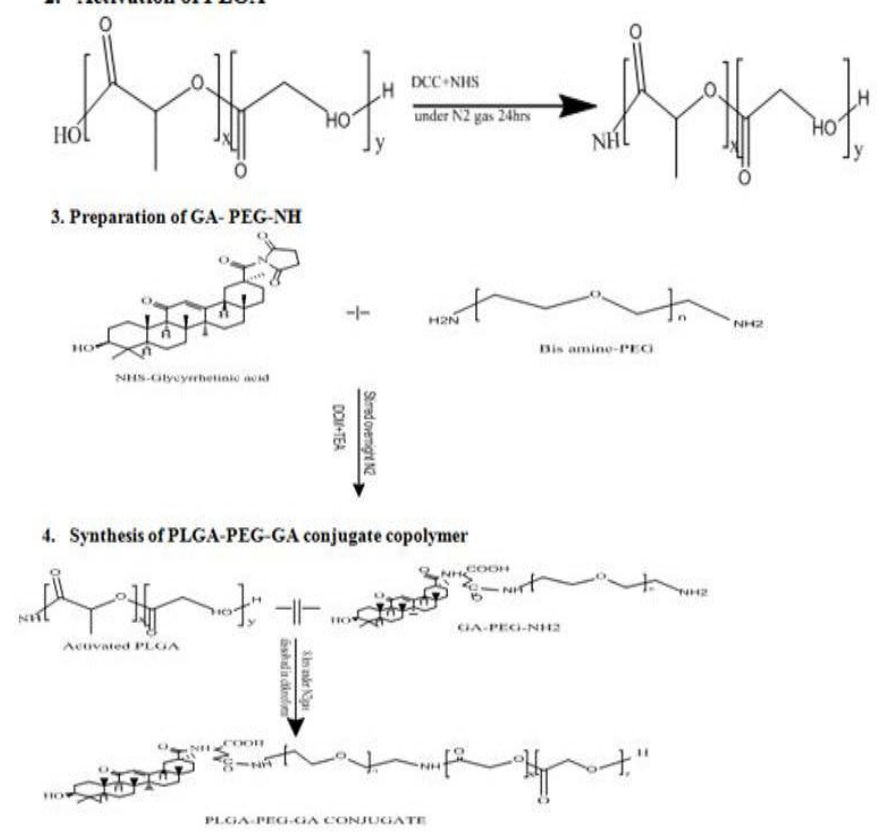

Figure 1. Final synthesis of GA-PEG- PLGA conjugate

\section{Activation of Glycyrrhetinic Acid (GA)}

Activation of glycyrrhetinic acid was performed according to the method described by Yogesh B. Patil with few modification, glycyrrhetinic acid (2.0 g) was dissolved in a mixture of anhydrous chloroform $(80 \mathrm{ml})$ and triethylamine (TEA $1.0 \mathrm{ml}$ ), and it was stirred for overnight in dark (15).
Then, the resultant solution was further stirred for $18 \mathrm{hrs}$, after mixing of dicyclohexylcarbodimide (DCC $1.0 \mathrm{~g}$ ) and Nhydroxysuccinamide (NHS, $0.56 \mathrm{~g}$ ) in it. Finally, side product dicyclohexylurea (DCU) was removed by filtration and solvents like chloroform and TEA were evaporated under vacuum. 


\section{Activation of PLGA Polymer}

Activation of PLGA (average molecular weight of $8000 \mathrm{Da}$ ) was carried out according to the method described by Haizheng Zhao briefly, $2 \mathrm{~g}$ of PLGA was activated by DCC (56.5 $\mathrm{mg}$ ) and NHS (31.5 mg) (molar ratio 1:1.1:1.1, respectively) in $5 \mathrm{ml}$ of dichloromethane (DCM) at room temperature under nitrogen gas for $24 \mathrm{~h}$ (16). The resultant was filtered to remove the by product dicyclohexylurea (DCU). After filtration, activated PLGA was precipitated by ice-cold diethyl ether and dried under vacuum.

\section{Preparation of GA-PEG-NH 2}

Glycyrrhetinic acid was conjugated with bis amine PEG $\left(\mathrm{NH}_{2}-\right.$ PEG-NH $\mathrm{N}_{2}$ ) according to the method described by Yogesh $\mathrm{B}$. Patil with slight modifications, Bis-amine-PEG $2.0 \mathrm{~g}$ (150 times more than NHS- GA acid, average molecular weight of $4000 \mathrm{Da})$ was dissolved in acetonitrile $(4.0 \mathrm{ml})$ (15). Methylene chloride $(2.0 \mathrm{ml})$ and TEA $(1.6 \mathrm{ml})$ were added to the above mixture and the solution was stirred for $1 \mathrm{~min}$ only. NHS- GA (500 mg) was added to the above mixture and stirred overnight under nitrogen. The reaction was blocked by the dropwise addition of diethyl ether (10-15 ml) to precipitate the polymer and separate the unreacted PEG. Then precipitated polymer was filtered and washed with diethyl ether. The untreated polymers were separated by dialysis (dialysis membrane MWCO, 4000).

\section{Synthesis of GA-PEG- PLGA conjugate copolymer}

The final synthesis was performed according to the method described by Haizheng Zhao with few modifications (16). Activated PLGA (1.0 g) and GA-PEG-NH2 (1.2 g) were added to $5 \mathrm{ml}$ of chloroform at room temperature for $8 \mathrm{hrs}$ under nitrogen gas. Then final product was precipitated in ice cold diethyl ether, and then this was further dissolved in chloroform and dialyzed for the removal of chloroform and unreacted polymers against deionized water over $72 \mathrm{hrs}$ (MWCO 12000). The further collected copolymer (GA-PEGPLGA) was dialyzed in chloroform (MWCO 14000) to remove the PLGA-PEG-PLGA copolymers. Then, the dialyzed copolymers were obtained by dialysis membrane against deionized water over $72 \mathrm{hrs}$, and also from vacuum dryer. Finally these were lyophilized, and final product was analyzed for conjugation through NMR spectrum. The conjugate was dissolved in $\mathrm{CDCI}_{3}$ and ${ }^{1} \mathrm{H}$ NMR spectra was recorded with employing NMR Spectrometer.

\subsection{Preparation of Polymeric Conjugate (GA-PEG-PLGA) Nanoparticles by Nanoprecipitation Method}

PLGA (50:50) nanoparticles are usually prepared by nanoprecipitation method. Nanoparticles were prepared according to the literature (17), with some modifications. GA-PEG-PLGA nanoparticles were formed spontaneously upon addition of various concentrations of GA-PEG-PLGA $(0.5,1,1.5,2,2.5$ and $20.0 \mathrm{mg} / \mathrm{ml})$ and homogenization under varying time and speed. Embelin-loaded nanoparticles were formed by incorporation of the solution into the GAPEG-PLGA solution containing $0.5 \mathrm{mg} / \mathrm{ml}$ embelin at the same ratio $(15,18)$. The solutions were stirred for 2 hours, followed by the complete removal of all organic solvents on the rotary evaporator. The nanoparticles are obtained as dispersed in the water solution $(19,20)$.

\section{Characterization of Nanoparticles}

\subsection{Particle Size Analysis}

Particle size and size distribution was determined by photon correlation spectroscopy using a Zeta sizer (Beckman Coulter Pvt. Ltd.). A dilute suspension of NPs $(20 \mu \mathrm{g} / \mathrm{ml})$ was prepared in deionized distilled water and measurements were taken in specific disposable cuvettes and recorded.

\subsection{Surface Morphology (TEM)}

The transmission electron microscopy of the Embelin loaded polymeric nanoparticles was analyzed by the transmittance electron microscopy by using the stain phosphotungstic acid. TEM is a method of probing the microstructure of rather delicate system such as micelles, liquid crystalline phases, vesicles, emulsions and also nanoparticles. A drop of particle dispersion was spread onto a 200 mesh copper grid coating and the droplets excess was removed with a filter paper. After 5 min, a drop of $2 \%$ phosphotungstic acid was placed onto the copper grid. The grid was dried at room temperature and observed by TEM.

\subsection{Entrapment Efficiency}

The entrapment efficiency of the Embelin loaded surface modified polymeric nanoparticles was carried out by centrifugation at low temperature and taking the absorption of supernatant at UV spectrophotometer at $292 \mathrm{~nm}$. The formula used for the calculation of the entrapment efficiency is given as:

$$
\text { Entrapment efficiency }=\frac{\text { Total amount o drug-Free amount of drug }}{\text { Total amount of drug }}
$$

\subsection{In-vitro Drug Release Studies}

In vitro drug release profile of entrapped drug from nanoparticles was studied at two different pH (1.2 and 6.8) using dialysis bag. In order to mimic gastric and intestinal environment the release of Embelin from Embelin loaded GA-PEG-PLGA nanoparticles were observed at SGF (pH 1.2) and SIF ( $\mathrm{pH}$ 6.8), respectively. Dialysis membrane was exposed to running water for 12 hours. In-vitro drug release studies were performed by dialysis bag method at rotation speed of $100 \mathrm{rpm}$. Acidic buffer solution (pH 1.2) was used as dissolution medium. Each dialysis bag (pore size: 12KD, Sigma Chemical Co., USA) was loaded with $20 \mathrm{mg}$ nanoparticles. Volume and temperature of dissolution medium were $200 \mathrm{ml}$, and $37.0 \pm 0.2{ }^{\circ} \mathrm{C}$ respectively. At predetermined time interval, samples $(5 \mathrm{ml})$ were withdrawn and replaced with same volume of fresh media for duration of 2 hours. The same procedure was repeated with basic buffer solution pH 6.8 for a time period of 120 hours along with sample withdrawal and replacement at predetermine time interval, filtered and assayed for drug content at 292 $\mathrm{nm}$ against blank using UV-Visible spectrophotometer.

\subsection{In-vivo Studies}

The formulation is prepared in order to target the drug directly to the liver. To investigate the beneficial potential of the final formulation (F12) in a hepatotoxicity experimental study in wistar rats was carried out. Wistar rats weighing 180-220 g of either sex were used. Animals were obtained from Central Animal House Facility of I.S.F. College of Pharmacy, Moga, Punjab, India. They were housed at ambient temperature $\left(21 \pm 10^{\circ} \mathrm{C}\right)$ and relative humidity $(55 \pm 5 \%)$ with fixed $12 \mathrm{~h}$ light/dark cycle. The experimental protocol was approved as ISFCP/IAEC/CPCSEA/2015/265 by Institutional Animal Ethical Committee (IAEC) as per the guidance of committee for the purpose of control and supervision of experiments on animals (CPCSEA). Hepatotoxicity was induced by administration of $40 \%$ alcohol at dose of $2 \mathrm{ml} / 100 \mathrm{~g} /$ day, p.o. for 21 days. Standard (Silymarin $25 \mathrm{mg} / \mathrm{kg} .$, p.o), Embelin ( $25 \mathrm{mg} / \mathrm{kg}$., p.o) and GAPEG-PLGA loaded Embelin (25 mg/kg., p.o) were suspended in $0.5 \%$ CMC (Carboxyl methylcellulose) solution and 
administered per orally for 21 days in respective groups, one and half hour prior to alcohol dosing. On 21st day all animals were sacrificed, blood was collected for assessment of liver function (SGOT, SGPT, ALP, and TB). The rats were divided into five different groups $(\mathrm{n}=5)$ as shown in Table 1.

Table 1. Optimization of polymer conjugate concentration

\begin{tabular}{|c|c|c|c|c|c|}
\hline Formulation & $\begin{array}{c}\text { Conjugate conc } \\
\text { (w/v) }\end{array}$ & $\begin{array}{c}\text { Homoginization Time } \\
(\mathbf{m i n})\end{array}$ & $\begin{array}{c}\text { Homoginization speed } \\
\mathbf{( r p m})\end{array}$ & $\begin{array}{c}\text { Particle Size } \\
\mathbf{( n m})\end{array}$ & PDI \\
\hline F1 & $\mathbf{0 . 5 \%}$ & $\mathbf{1 0}$ & $\mathbf{4 0 0 0}$ & $\mathbf{2 2 3 . 3 \pm 1 . 1}$ & $\mathbf{0 . 1 8 8}$ \\
\hline F2 & $1 \%$ & 10 & 4000 & $291 \pm 1.4$ & 0.261 \\
\hline F3 & $1.5 \%$ & 10 & 4000 & $355.1 \pm 1.2$ & 0.209 \\
\hline F4 & $2 \%$ & 10 & 4000 & $438.2 \pm 0.9$ & 0.192 \\
\hline F5 & $2.5 \%$ & 10 & 4000 & $571.3 \pm 1.3$ & 0.241 \\
\hline F6 & $3 \%$ & 10 & & $495.2 \pm 0.8$ & 0.267 \\
\hline
\end{tabular}

Number of groups $=5$; Total no. of rats $=25$ ( 5 Rats in each group); Dose $=25 \mathrm{mg} / \mathrm{kg} / \mathrm{per}$ oral

\subsection{Hepatotoxicity Study of Final Polymeric Conjugate \& Optimized Drug Loaded Nanoparticulate Formulation.}

Since the formulation is prepared in order to target the drug (Embelin) directly to the liver, Hence it becomes very important to check the hepatotoxicity of the final prepared polymeric conjugate as well as the drug loaded final nanoparticulate formulation. Different tests like determination of SGOT, SGPT and ALP and TB level study of the liver were done in order to check the liver toxicity of the final polymeric conjugate and final nanoparticulate formulation.

\subsubsection{Aspartate transaminase (AST/SGOT)}

Oral administration of $40 \%$ alcohol $(2 \mathrm{ml} / 100 \mathrm{~g}$ ) treatment caused a significant increased SGOT level. AST (SGOT) catalyses the transfer of amino group between L-Aspartate and $\alpha$ - Ketoglutarate to form oxaloacetate and glutamate. The oxaloacetate formed reacts with NADH in the presence of malate dehydrogenase to form NAD. The rate of oxidation of NADH to NAD is measured as a decrease in absorbance is proportional to the AST (21). The surface modified Embelin GA-PEG-PLGA NPs protect the liver and reduce the increase level of the SGOT in the liver enzyme.

\subsubsection{Alanine transaminase (ALT / SGPT)}

Oral administration of $40 \%$ alcohol $(2 \mathrm{ml} / 100 \mathrm{~g}$ ) treatment caused a significant increased SGPT level. ALT (SGPT) catalyses the transfer of amino group between L-alanine and $\alpha$ - ketoglutarate to form pyruvate and glutamate. The pyruvate formed reacts with NADH in the presence of lactate dehydrogenase to form NAD. The rate of oxidation of NADH to NAD is measured as a decrease in absorbance which is proportional to the ALT (21). The surface modified Embelin GA-PEG-PLGA NPs protect the liver and reduce the increase level of the SGPT in the liver enzyme.

\subsubsection{Alkaline phosphatase (ALP)}

Oral administration of $40 \%$ alcohol ( $2 \mathrm{ml} / 100 \mathrm{~g}$ ) treatment caused a significant increased ALP level. ALP in an alkaline $\mathrm{pH}$, hydrolyses p-nitrophenylphosphate to form $\mathrm{p}$ nitrophenol and phosphate; the rate of formation of $p$ nitrophenol is measured as an increase in absorbance which is proportional to the ALP activity in the sample (22). The surface modified Embelin GA-PEG-PLGA NPs protect the liver and reduce the increase level of the ALP in the liver enzyme.

\subsubsection{Total bilirubin level (TB)}

Oral administration of $40 \%$ alcohol $(2 \mathrm{ml} / 100 \mathrm{~g})$ treatment caused a significant increased TB level. Bilirubin reacts with diazotized sulphanilic acid to form a coloured azobilirubin compound. The unconjugated bilirubin couples with the sulphanilic acid in the presence of a caffeine-benzoate accelerator. The intensity of the colour formed is directly proportional to the amount of bilirubin present in the sample (23). Embelin GA-PEG-PLGA NPs protect the liver and reduce the increase level of the TB in the liver enzyme.

\subsection{Cellular Uptake Study}

Cell uptake study of the formulation was performed for qualitative estimation of the uptake of drug by the liver cells. Cell uptake study of Embelin drug loaded surface modified (PLGA-PEG-GA) conjugated nanoparticles was performed on HepG2 cells by using rhodamine dye (Fluorecent dye) [20]. The cell culture was freshly prepared by sub culturing the cells using Dulbecco's Minimum Essential Medium (DMEM). Formulation was incubated with the cells using 6 well culture plates for 2 hours. Plain cells in media served as positive control. Images of control and test sample were taken using Fluoroscent Microscope (Olympus, CK X 41).

\subsection{In-vivo Pharmacokinetic and Liver Homgenate Studies Bioanalytical Method Development by HPLC for Pharmacokinetic Studies}

Optimized analytical method was already developed which is a selective and sensitive method for analysis of Embelin on HPLC using UV detection by PDA (Photo diode array) detector. A mobile phase of potassium dihydrogen orthophosphate $\left(\mathrm{KH}_{2} \mathrm{PO}_{4}\right)$ buffer of $\mathrm{pH} 7.4$ was investigated to develop a fully validated assay method using UV detection. The full validation of this method was performed on an HPLC-PDA system.

\section{RESULTS AND DISCUSSION}

\subsection{Optimization of Embelin loaded polymeric conjugated nanoparticles}

The prepared formulations were evaluated to get an optimized formulation. The different parameters were optimized such as polymer drug concentration, homogenization time and speed. Particle size is the most important parameter for target drug delivery. Smaller sized particles have larger surface area. The formulation was optimized according to their particle size, and polydispersity 
index. The optimization of finally prepared polymeric conjugated nanoparticles is optimized upto F1 to F6, however F1 is optimized polymeric conjugated concentration and which is shown in Table 2. As we increase the polymer concentration the particle size of nanoparticles increases, it may be because of increase in the uniform coating of polymer solution over the nanoparticle systems. It was seen that after certain limit with the increase in the homogenization time and speed the size of nanoparticles increases this is because of the increase in the kinetic energy of the nanoparticles leading to aggregation in particles. The time and speed in homogenization are optimized in formulation F7 to F10 and the optimized formulation is F9 which is shown in Table 2. The optimization of drug concentrations were evaluated for entrapment efficiency and the formulation with maximum efficiency was selected as the final formulation (F9) is given in the Table 2. It was seen that with the increase in drug concentration the entrapment efficiency of nanoparticles increase till the ratio of polymer and drug was 2:1. With further increase in the drug concentration the size of nanoparticles increases and entrapment efficiency decreases and the final optimized formulation is F12.

Table 2: Optimization of Homoginization Time (min)

\begin{tabular}{|c|c|c|c|c|c|}
\hline Formulation & $\begin{array}{c}\text { Conjugate conc } \\
(\mathbf{w} / \mathbf{v})\end{array}$ & $\begin{array}{c}\text { Homoginization Time } \\
(\mathbf{m i n})\end{array}$ & $\begin{array}{c}\text { Homoginization speed } \\
\text { (rpm) }\end{array}$ & $\begin{array}{c}\text { Particle Size } \\
\text { (nm) }\end{array}$ & PDI \\
\hline F7 & $0.5 \%$ & 10 & 4000 & $442.5 \pm 1.7$ & 0.207 \\
\hline F8 & $0.5 \%$ & 15 & 4000 & $338.2 \pm 1.6$ & 0.250 \\
\hline F9 & $\mathbf{0 . 5 \%}$ & $\mathbf{2 0}$ & $\mathbf{4 0 0 0}$ & $\mathbf{2 1 2 . 9 \pm 1 . 1}$ & $\mathbf{0 . 1 3 2}$ \\
\hline F10 & $0.5 \%$ & 25 & 4000 & $378.1 \pm 1.6$ & 0.175 \\
\hline
\end{tabular}

\subsection{Characterization of optimized formulation of GA- PEG-PLGA Nanoparticles}

Surface modified (GA-PEG-PLGA) NPs were prepared by the nanoprecipitation method $(15,24)$. The copolymer (GA-PEGPLGA) was synthesized and the final product of copolymer confirmed by $1 \mathrm{H}$ NMR spectroscopy results. The $1 \mathrm{H}$ NMR spectrum (Figure 2) shows proton peaks at $3.5 \mathrm{ppm}$ for $\mathrm{NH}_{2}$ and $\mathrm{NH}$, and $\sim 1.0 \mathrm{ppm}$ for $\mathrm{CH}_{2}$ which confirms presence of PEG, and hydrogen of the methine group of the lactic acid unit of the PLGA copolymer resonated at $5.3 \mathrm{ppm}$, whereas those of the methylene group of the glycolic acid unit appeared at $4.8 \mathrm{ppm}$ and $\sim 1.5 \mathrm{ppm}$ of $\mathrm{CH}_{3}$ confirms presence of PLGA. The peaks shown in the Figure 2, also matched with the peaks of PEG and PLGA as described by Yadav, Zhao and
Patil $(15,16,25)$. In Figure-2 the additional proton peaks at 6.80 and $7.21 \mathrm{ppm}$ exhibit the presence of $\mathrm{C}-\mathrm{NH}$, and 1benzene $\mathrm{CH}$, respectively which confirm the presence of GAPEG, whereas other proton peaks were same as Figure 3 which confirms the presence of PEG and PLGA. The peaks shown in the Figure 3, also matched with the peaks of PLGA, PEG and GA as described by Zhao and Patil $(15,16)$. So it can be concluded that both the conjugation has been successfully developed. These prepared surface modified GA-PEG-PLGA NPs were characterized for shape and surface morphology, particle size, drug entrapment efficiency. The GA-PEG-PLGA NPs were obtained in an average size of $316 \pm 1.9 \mathrm{~nm}$ with an entrapment efficiency of $96.3 \pm 3.1 \%$ and the particle size was analyzed by Zeta sizer.

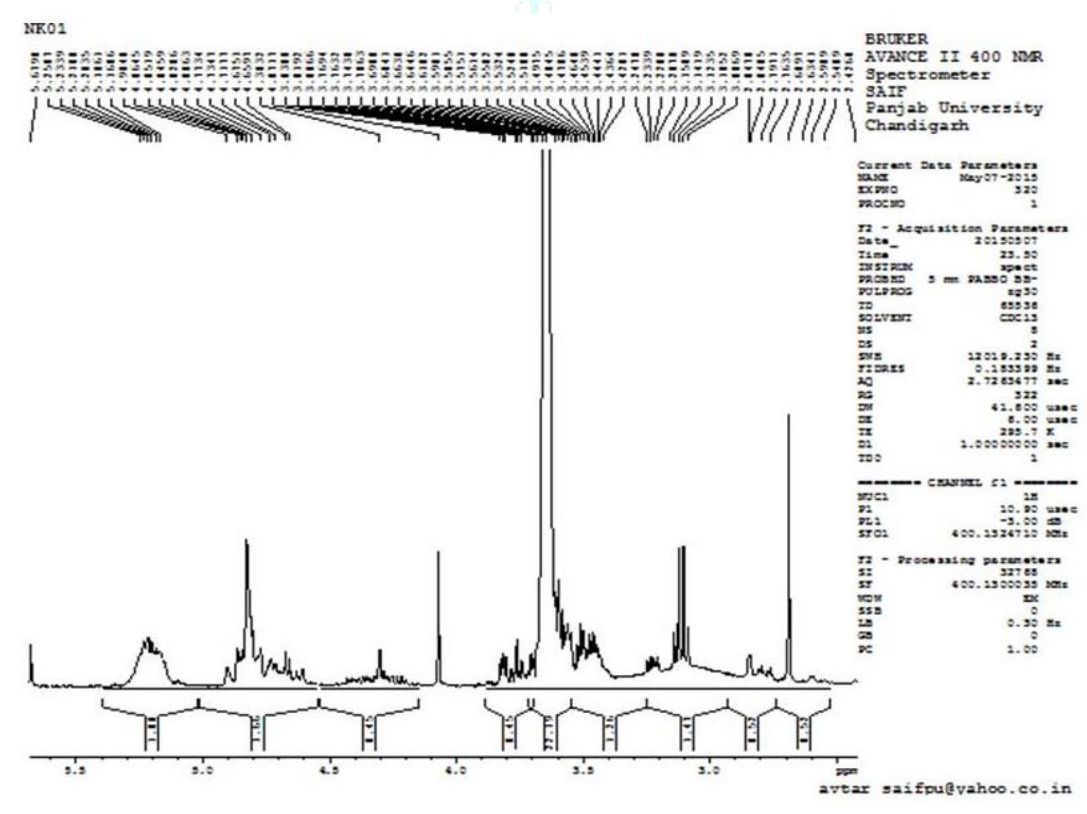

Figure 2. 1H NMR spectrum synthesis of GA-PEG- PLGA conjugate

The optimized formulation was visualized under transmission electron microscopy (TEM). Figure 3 shows TEM images of optimized NPs. TEM images revealed that NPs formed were small in size and were almost spherical in shape. The TEM photomicrograph exhibits NPs indicating that optimized formulation of GA-PEG-PLGA nanoparticles initially confirms burst release and $80 \pm 4.5 \%$ drug release in $120 \mathrm{~h}$. This could also be due to complete hindrance of core through PEG over the surface of NPs. Finally more sustained release of drug from GA-PEG-PLGA NPs also increases the effectiveness of carrier for the treatment of hepatotoxicity. 


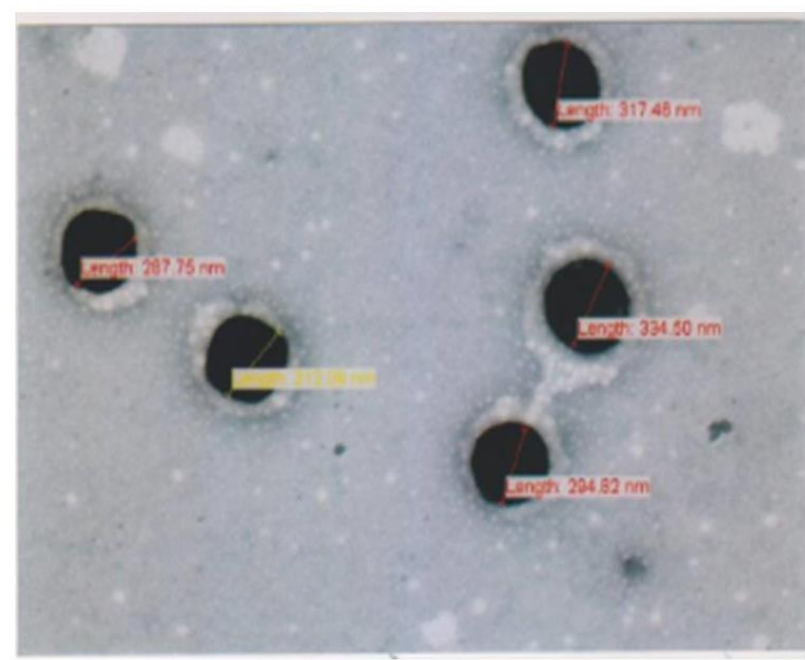

Figure 3. TEM images of embelin loaded GA-PEG- PLGA NPs.

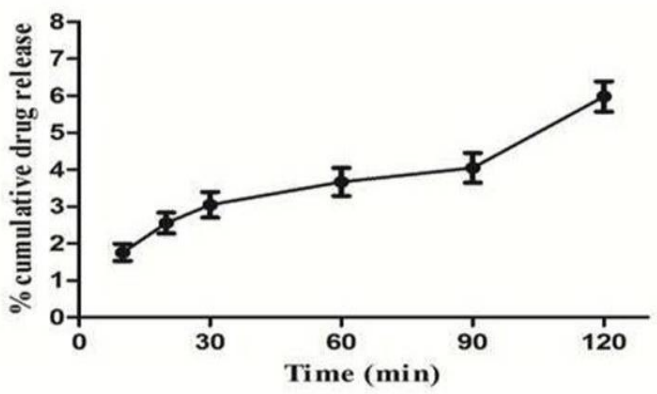

\subsection{In-vitro Drug Release Studies}

In-vitro drug release was performed using dialysis bag at $\mathrm{pH}$ 1.2 and 6.8. In order to mimic the conditions of our system, the release study is performed at gastric $\mathrm{pH}$ (SGF pH 1.2) and intestinal $\mathrm{pH}$ (SIF pH 6.8) separately. Figure 4 shows the in vitro drug release from surface modified (PLGA-PEG-GA) NPs. In-vitro cumulative release was carried out for optimized formulation ((PLGA-PEG-GA) nanoparticles) using Embelin at $\mathrm{pH} 1.2$ and 6.8 and it was observed that the drug release was found to be $6.04 \%$ for 2 hours at SGF (pH 1.2) and was $80 \pm 4.5 \%$ at SIF pH 6.8 as compare to $\mathrm{pH} 1.2$. In-vitro release profile establishes the enteric coating of the prepared formulation. Polyanionic nature of the polymer remains nonprotonated in the gastric $\mathrm{pH}$. Thereby inhibits the release of encapsulated therapeutics whereas intestinal $\mathrm{pH}$ induces the protonation of the coating material causes a controlled release of encapsulated material. Controlled release is further attributed to the physiochemical behaviour of polymer conjugate where the conjugate remains unprotonated in intestinal $\mathrm{pH}$, thus restrict the free access to dissolution medium, leads to controlled diffiusion of drug.

Figure 4. In- vitro drug release simulated gastric fluid 1.2 and PBS 7.4

\subsection{Cellular Uptake Study}

Cell uptake studies of the formulation were performed for the qualitative estimation of uptake of the nanoparticles by

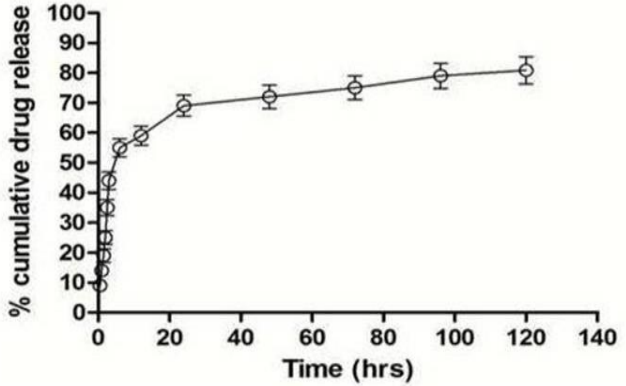

liver cells. Image of the cells taken after 12 hours of incubation with dispersion of drug loaded nanoparticles is shown in Figure 5.
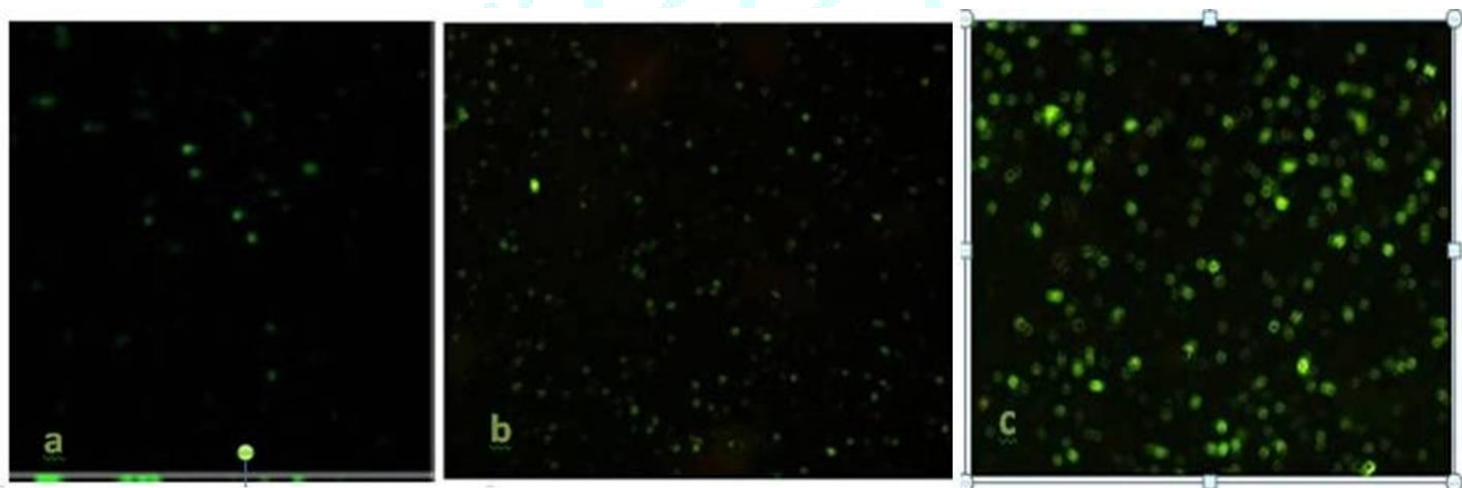

Figure 5. Representation of the Cellular uptake (a) plain Embelin (b) standard drug Silymarin (c) Embelin loaded GAPEG-PLGA NPs.

It is clearly revealed from the images that the surface modified Embelin loaded (GA-PEG-PLGA) NPs has the maximum cellular uptake as compared to plain drug have less cellular uptake in comparisons. A high cellular uptake of polymer conjugated nanoparticles established the proposed hypothesis. In that the presence of GA improves the cellular efficiency of developed formulation for hepatic cells. Cell uptake study further confirms that receptor mediated endocytosis is a key mechanism for cellular uptake of developed formulation.

\subsection{In-vivo Drug Concentration}

HPLC method was developed in human plasma for estimation of Embelin. Plasma drug concentration-time profile of surface modified Embelin loaded GA-PEG-PLGA nanoparticles (F12), administered orally in wistar rats is 
present in Table 3 represents various pharmacokinetic parameters. From the given results it was observed that the developed optimized formulation showed increased plasma concentration $(61.49 \mu \mathrm{g} / \mathrm{ml})$ and $\mathrm{T}_{1 / 2}(41.44 \mathrm{hrs})$ than the plain drug and significant to the standard drug. Further the plasma concentration takes longer time to reach its maximum value in case of F12 formulation followed by plain drug showing sustained release in case of GA-PEG-PLGA formulation. This could be attributed to preferential localization of polymer conjugate nanoparticle in liver.
Polymer conjugate showed $25 \%$ rise in $\mathrm{C}_{\max }$ value which could be attributed to increase bioavailability of developed formulation attributed to nanoparticles dispersion, controlled release mechanism and retention of the developed formulation near the absorption window. Further a higher $\mathrm{T}_{\max }$ in developed formulation is associated with its high affinity for hepatic cell lines. Further a higher $\mathrm{T}_{\max }$ in developed formulation is associated with its high affinity for hepatic cell lines.

Table 3. Plasma pharmacokinetic parameters of different formulation

\begin{tabular}{|c|c|c|c|}
\hline Parameters & Silymarin & $\begin{array}{c}\text { Glycyrrhetinic acid -PEG-PLGA } \\
\text { Embelin nanoparticles }\end{array}$ & Plain drug solution \\
\hline $\mathrm{C}_{\max }(\mu \mathrm{g} / \mathrm{ml})$ & 63.23 & 61.49 & 43.55 \\
\hline $\mathrm{T}_{\max }(\mathrm{hour})$ & 32 & 32 & 24 \\
\hline $\mathrm{T}_{1} / 2(\mathrm{hour})$ & 46.58 & 41.44 & 19.5 \\
\hline $\mathrm{AUC}$ total $(\mu \mathrm{g} / \mathrm{ml})$ & 5896.5 & 5430.1 & 2764 \\
\hline $\mathrm{MRT}$ & 77.72 & 71.93 & 50.59 \\
\hline
\end{tabular}

Further the localization of receptor along the sinusoid cell lines improves hepatic permeability and retention of the developed formulation in the liver as shown in Table 4. For this all animals were sacrificed on $21^{\text {st }}$ day under light ether anesthesia; blood samples were collected and allowed to clot for 30 minutes at room temperature, then centrifuged at $3000 \mathrm{rpm}$ for 20 minutes. Serum was separated and stored at $-20^{\circ} \mathrm{C}$ until biochemical estimation for liver profile.

Table 4. Represents amount of drug in liver with respect to time

\begin{tabular}{|c|c|c|c|}
\hline Time (hour) & Silymarin & GA-PEG-PLGA & Plain drug solution \\
\hline 16 & $42.26 \pm 2.34$ & $40.24 \pm 2.81$ & $15.87 \pm 2.1$ \\
\hline 32 & $63.38 \pm 2.78$ & $61.43 \pm 2.45$ & $28.09 \pm 2.37$ \\
\hline 72 & $30.51 \pm 2.9$ & $28.03 \pm 2.31$ & $12.87 \pm 2.04$ \\
\hline 120 & $16.05 \pm 2.76$ & $14.24 \pm 2.21$ & $4.34 \pm 2.43$ \\
\hline
\end{tabular}

Figure 6 (a) depicts graphical representation of plasma concentration of Embelin at each time interval point which indicates that highest plasma concentration of Embelin at each time interval point which indicates that highest plasma concentration was obtained from F12 nanoparticles formulation which is comparable and significant to marketed Silymarin $(\mathrm{p}<0.05)$. From Figure 6 (b) it is concluded that the amount of drug uptake in the liver is more in GA-PEG-PLGA nanoparticles as compared to plain drug and significant to the standard drug Silymarin $(\mathrm{p}<0.05)$. The final formulation F12 nanoparticles increase the bioavailability of drug in liver by 2.0 folds more than plain drug. So it can be attributed that Glycyrrhetinic acid has the ligand properties for the receptors presents in the liver, so can be used for liver targeting, as well as it is efficient against hepatotoxicity.

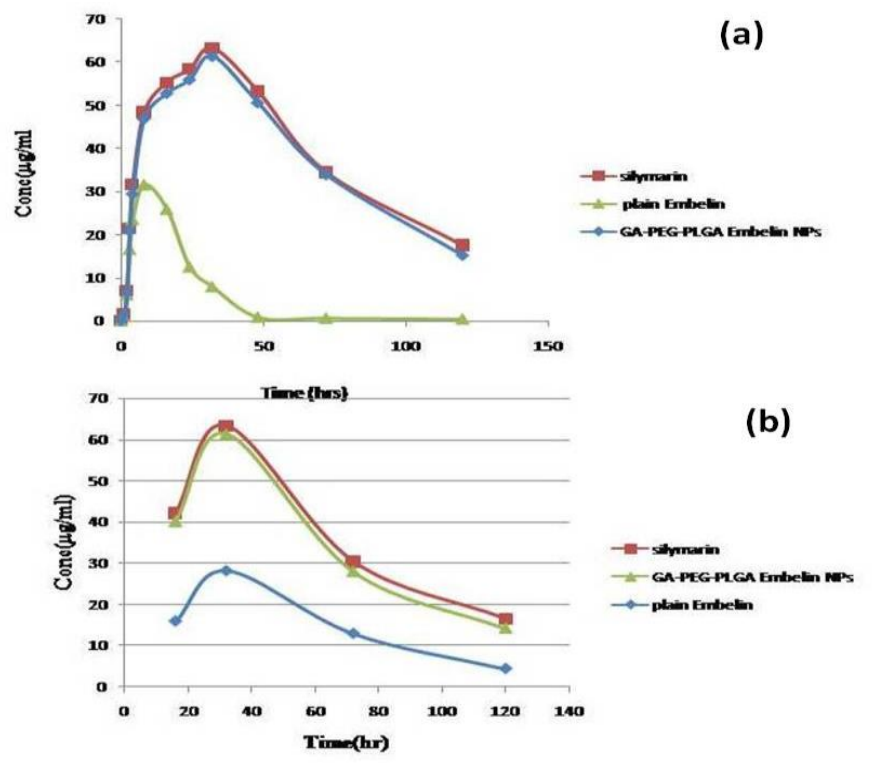

Figure 6. Representation of the (a) plasma concentration of Embelin (b) Embelin concentration in liver 


\subsection{Hepatotoxicity Study of Final Formulation}

Effect of surface modified (GA-PEG-PLGA) Embelin loaded nanoparticles was studied on liver enzymes such as Serum glutamic oxaloacetic transaminase (SGOT), Serum glutamic pyruvic transaminase (SGPT), Total bilirubin (TB) and Alkaline phosphatase (ALP) in $40 \%$ alcohol induced rats suffering from hepatotoxicity. Oral administration of $40 \%$ alcohol $(2 \mathrm{ml} / 100 \mathrm{~g})$ treatment caused a significant increase liver enzymes level on 21st day as compared to control treated group. Treatment with standard drug silymarin (25 $\mathrm{mg} / \mathrm{kg}$ ), plain Embelin $(25 \mathrm{mg} / \mathrm{kg}$ ), surface modified (GAPEG-PLGA) nanoparticles $(25 \mathrm{mg} / \mathrm{kg}$ ) showed significant decrease in liver enzymes level as compared to $40 \%$ alcohol treated group. However effect of surface modified Embelin loaded GA-PEG-PLGA NPs was more significant in comparison to Plain Embelin, that may be due to selective ligand targeting of Embelin loaded GA-PEG-PLGA NPs to the liver which is shown in Figure $7 \mathrm{a}-7 \mathrm{~d}$, so that it can be used for liver targeting, as well as it is efficient against hepatotoxicity. a
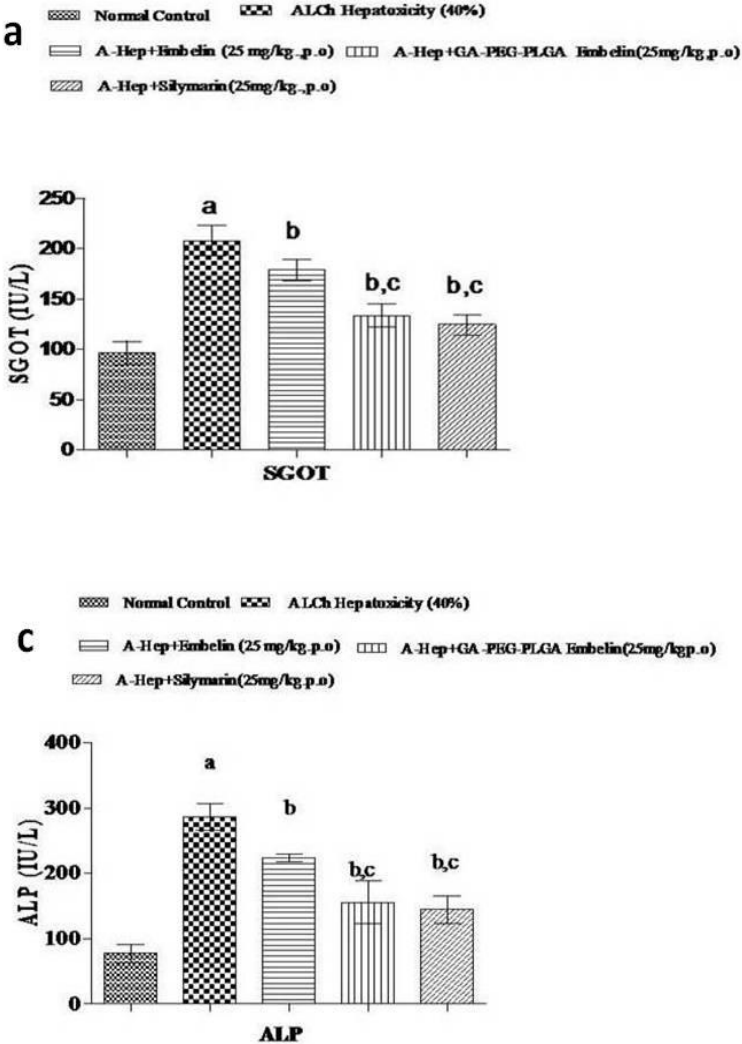

b
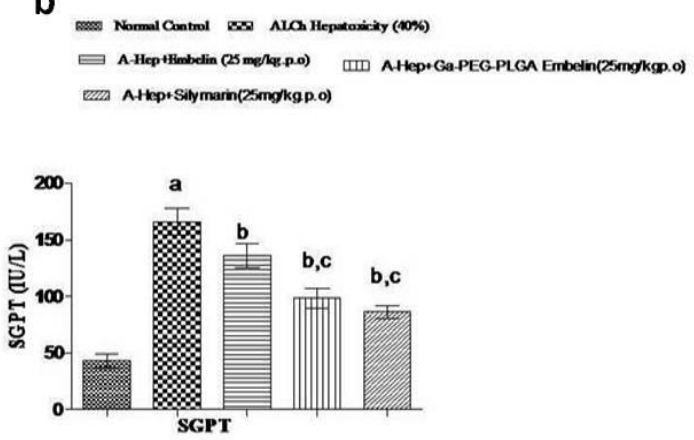

d
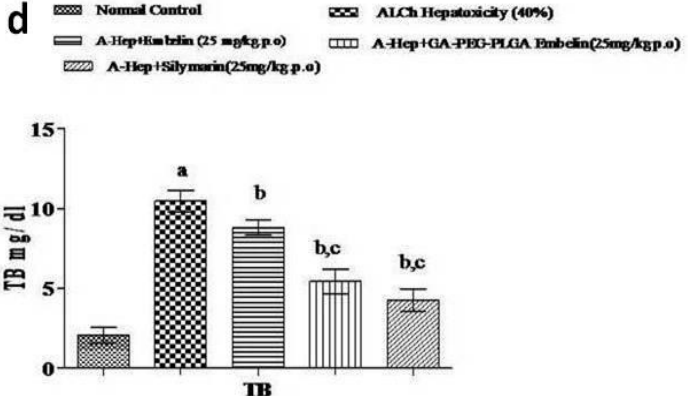

Figure 7. Effect of Embelin and Embelin loaded (GA-PEG-PLGA) NPs in alcohol induced change in (a) SGOT (b) SGPT (c) ALP (d) TB

\section{CONCLUSION}

This work report that GA-PEG-PLGA nanoparticles can be used as the carrier of Embelin for hepatotoxicity for liver targeting in effective management of alcohol induced hepatotoxicity. Liver targeted nanoparticles (GA-PEG-PLGA) composed of GA and PLGA could be prepared conveniently by the nanoprecipitation method. GA-PEG-PLGA NPs are nearly spherical of $316 \mathrm{~nm}$ in diameter with homogeneous structure and smooth surfaces. A significant decline in the percent drug release of Embelin from GA-PEG-PLGA NPs SIF (pH 6.8) indicated that coupling of glycyrrhetinic acid slows down the release of drug from the NPs and thereby imparts a sustained release nature. When Embelin given by oral route, blood circulation distributed it to all the organs, this is remarkably higher than that of the nanoparticles with the GA in liver. Moreover, the in vitro cell uptake results showed that the introduction of GA to the nanoparticles could significantly increase the affinity to hepatictoxicity with approximately an improvement in cellular uptake. Surface modified Embelin loaded (GA-PEG-PLGA) NPs significantly reduced the levels of SGOT, SGPT, ALP and TB in comparison to Alcholic-Hepatotoxic group rats. More significant improvement in liver function by Surface modified Embelin loaded (GA-PEG-PLGA) NPs in comparison to plain embelin may be attributed to increase in targeting of liver due to interaction of GA with its liver GA- receptors. Thus it is concluded that surface modification of embelin loaded (GAPEG-PLGA) NPs increases the liver targeting and have beneficial potential in alcoholic induced hepatoxocity.

\section{Acknowledgement}

Authors are thankful to Mr. Praveen Garg, Chairman, ISF College of Pharmacy, Moga, Punjab, for his continuous support and encouragement.

\section{Declaration of interest}

The authors report no declarations of interest. The authors alone are responsible for the content and writing of the paper.

Gupta AK, Dhakar RC, Siddiqui AW, Maurya SD, Interpenetrating polymeric network hydrogel for stomachspecific drug delivery of clarithromycin: Preparation and evaluation, Asian Journal of Pharmaceutics, 2010; 4(4):184189 


\section{REFERENCES}

1. Mishra N, Yadav NP, Rai VK, Sinha P, Yadav KS, Jain S, et al. Efficient hepatic delivery of drugs: novel strategies and their significance. BioMed research international. 2013;2013.

2. Moghimi SM, Hunter AC, Murray JC. Long-circulating and targetspecific nanoparticles: theory to practice. Pharmacological reviews. 2001;53(2):283-318.

3. Brannon-Peppas L, Blanchette JO. Nanoparticle and targeted systems for cancer therapy. Advanced drug delivery reviews. 2012;64:206-12.

4. Gu FX, Karnik R, Wang AZ, Alexis F, Levy-Nissenbaum E, Hong S, et al. Targeted nanoparticles for cancer therapy. Nano today. 2007;2(3):14-21.

5. Poojari R, Gupta S, Maru G, Khade B, Bhagwat S. Chemopreventive and hepatoprotective effects of embelin on $\mathrm{N}$ nitrosodiethylamine and carbon tetrachloride induced preneoplasia and toxicity in rat liver. Asian Pac J Cancer Prev. 2010;11(4):1015-20.

6. Kothavade R, Joglekar S, Barodavalla S. Protective effect of an indigenous drug livomyn on ketoconazole induced hepatotoxicity. Indian journal of pharmaceutical sciences. 1996;58(4):142.

7. Gupta O, Ali MM, Ray GB, Atal C. Some pharmacological investigations of embelin and its semisynthetic derivatives. Indian journal of physiology and pharmacology. 1976;21(1):319.

8. Ghosh N, Ghosh R, Mandal V, Mandal SC. Recent advances in herbal medicine for treatment of liver diseases. Pharmaceutical Biology. 2011;49(9):970-88.

9. Singh D, Singh R, Singh P, Gupta RS. Effects of embelin on lipid peroxidation and free radical scavenging activity against liver damage in rats. Basic \& clinical pharmacology \& toxicology. 2009;105(4):243-8.

10. Kawakami S, Munakata C, Fumoto S, Yamashita F, Hashida M. Novel galactosylated liposomes for hepatocyte-selective targeting of lipophilic drugs. Journal of pharmaceutical sciences. 2001;90(2):105-13.

11. Sato A, Kawakami S, Yamada M, Yamashita F, Hashida M. Enhanced gene transfection in macrophages using mannosylated cationic liposome-polyethylenimine-plasmid DNA complexes. Journal of drug targeting. 2001;9(3):201-7.

12. Huang W, Wang W, Wang $P$, Tian $Q$, Zhang $C$, Wang $C$, et al. Glycyrrhetinic acid-modified poly (ethylene glycol)-b-poly $(\gamma$ - benzyl l-glutamate) micelles for liver targeting therapy. Acta biomaterialia. 2010;6(10):3927-35.

13. Onishi H, Takahashi H, Machida Y. Preparation and evaluation of glycyrrhetic acid-containing microparticles as an anti-hepatotoxic system. Drug development research. 2005;66(3):189-99.

14. Zhi Y, Huang W, Tian Q, Zhang C, Han L, Liu T, et al. Glycyrrhetinic acid-mediated nanoparticles of hepatic targeted drug delivery system, process for preparing the same and use thereof. Google Patents; 2015.

15. Patil YB, Toti US, Khdair A, Ma L, Panyam J. Single-step surface functionalization of polymeric nanoparticles for targeted drug delivery. Biomaterials. 2009;30(5):859-66.

16. Zhao H, Yung LYL. Selectivity of folate conjugated polymer micelles against different tumor cells. International journal of pharmaceutics. 2008;349(1):256-68.

17. Nordström P. Formation of polymeric nanoparticles encapsulating and releasing a new hydrophobic cancer drug. 2011.

18. Kara A, Ozturk N, Sarisozen C, Vural I. Investigation of formulation parameters of plga nanoparticles prepared by nanoprecipitation technique. 2014.

19. Hornig S, Heinze T, Becer CR, Schubert US. Synthetic polymeric nanoparticles by nanoprecipitation. Journal of Materials Chemistry. 2009;19(23):3838-40.

20. Zhang L, Zhang L. Lipid-polymer hybrid nanoparticles: synthesis, characterization and applications. Nano Life. 2010;1(01n02):163-73.

21. Bradley D, Maynard J, Emery G, Webster H. Transaminase activities in serum of long-term hemodialysis patients. Clinical chemistry. 1972;18(11):1442-.

22. Lieberman D, Phillips D. " Isolated" elevation of alkaline phosphatase: significance in hospitalized patients. Journal of clinical gastroenterology. 1990;12(4):415-9.

23. Jendrassik L, Grof P. Simplified photometric methods for the determination of the blood bilirubin. Biochem $\mathrm{Z}$. 1938;297(81):b5.

24. Song X, Zhao Y, Hou S, Xu F, Zhao R, He J, et al. Dual agents loaded PLGA nanoparticles: systematic study of particle size and drug entrapment efficiency. European journal of pharmaceutics and biopharmaceutics. 2008;69(2):445-53.

25. Yadav AK, Mishra P, Mishra AK, Mishra P, Jain S, Agrawal GP. Development and characterization of hyaluronic acid-anchored PLGA nanoparticulate carriers of doxorubicin. Nanomedicine: Nanotechnology, Biology and Medicine. 2007;3(4):246-57. 